\title{
Combined Experimental and Monte-Carlo Ray-Tracing Approach for Optimizing Light Extraction in LED COB Modules
}

\author{
Marianne Consonni, Julien Routin, Anthony Piveteau, and Adrien Gasse
}

CEA-Leti, Minatec Campus, 17 rue des Martyrs, 38054 Grenoble Cedex 9, France

Correspondence should be addressed to Marianne Consonni; marianne.consonni@cea.fr

Received 6 May 2013; Accepted 26 June 2013

Academic Editors: L. R. P. Kassab and Y. S. Kivshar

Copyright ( 2013 Marianne Consonni et al. This is an open access article distributed under the Creative Commons Attribution License, which permits unrestricted use, distribution, and reproduction in any medium, provided the original work is properly cited.

High-power light-emitting diodes (LEDs) for lighting applications require a high-efficient packaging to optimize their performances. Due to its high thermal dissipation potential, the chip-on-board (COB) technology is widely used for developing high-power lighting sources. In order to optimize the optical properties of such sources and to propose high optically efficient encapsulation geometry, ray-tracing simulations have been performed. The impact of the shape and volume of the silicone encapsulation on the light extraction and on the intensity distribution of the module was derived. Then, simulation results were correlated with experimental measurements on blue light-emitting COB sources. It is shown that a nearly hemispherical encapsulation with a minimal volume of 5 to $10 \mathrm{~mm}^{3}$ for a $1 \mathrm{~mm}^{2}$ LED die is the optimal configuration regarding both the light extraction and the intensity distribution.

\section{Introduction}

High-power light-emitting diodes (LEDs) have attracted a lot of interest in the recent years due to their low-power consumption and long lifetime. However, the integration of these light sources for general lighting applications will require high-efficient packaging with low thermal resistance and compact size [1]. Thus, due to its compactness and its high thermal dissipation potential, the chip-on-board (COB) technology is one of the favourite candidates for developing customized and thermal-efficient LEDs [2].

Beyond the thermal efficiency, one of the key performance criteria of a LED light source is its optical efficiency. This efficiency comprises several components [3]. The first one is the "internal quantum efficiency". It is defined as the ratio between the number of photons emitted from the active region per second and the number of electrons injected into the LED per second. It describes the LED junction performance.

In this paper, we focus on the second component of this efficiency: the "light extraction efficiency". It is defined as the ratio between the number of photons emitted into free space per second and the number of photons emitted from the active region per second. At the die level, this efficiency is limited by the total internal reflexions which appear at the interface between the semiconductor material and the air due to their refractive index mismatch. It may be improved either by structuring the die surface $[4,5]$ and/or by packaging the die with dome-shaped encapsulants with a high refractive index [6]. At the package level, the extraction is limited by the losses which may occur during light propagation within the package. A lot of work has been done to reduce these losses and to optimize the light extraction of a packaged LED. This included adjusting the encapsulation refractive index [7], inserting a reflective part around the light-emitting element $[8,9]$, or optimizing the exit surface shape of the package $[9,10]$.

However, in a COB module, an important point is to keep a simple realization process, which allows both cost reduction and performance. In order to reach this target and to optimize the module performances, the parameters which are easier to modify are the encapsulation shape and the volume. Several studies have already been performed to evaluate the impact of the encapsulation on the light extraction [11, 12]. Nevertheless, to our knowledge, these 


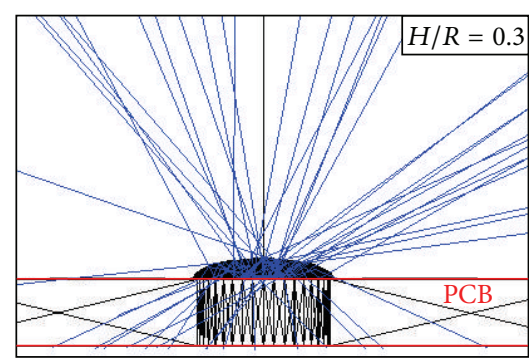

(a)

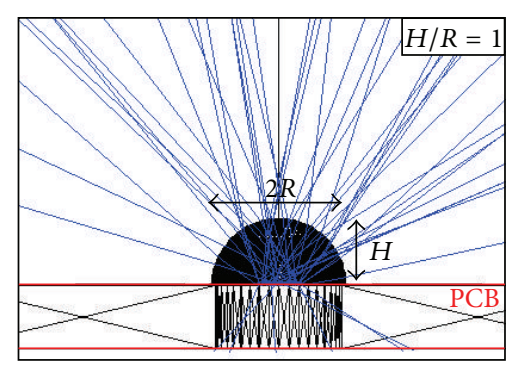

(b)

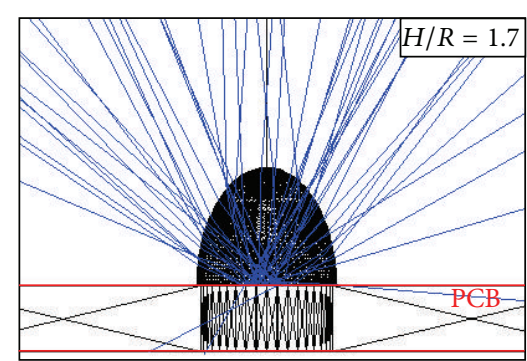

(c)

FIGURE 1: Cross-sections of the geometries which have been modelled for evaluating the impact of the encapsulation shape and size on a LED COB performance. (a) Nearly flat encapsulation, with an aspect ratio $H / R$ of 0.3 ; (b) hemispherical encapsulation; (c) oblong encapsulation, with an aspect ratio $H / R=1.7$.

works stayed at the theoretical stage, and they were not demonstrated experimentally.

That is why we propose in this paper to combine both a ray-tracing and an experimental approach for optimizing the light extraction and intensity distribution of a LED COB module. Thanks to the Monte-Carlo ray-tracing simulations, we have determined the encapsulation shape and the volume allowing to maximize light extraction. Then, the originality of this study is that these results have been correlated with experimental measurements done on COB samples, which were made from a $1 \mathrm{~mm}^{2}$ LED die encapsulated with silicone. The experimental results are found to be in good agreement with the simulations.

\section{Simulations}

In order to determine the parameters which are critical for the LED COB encapsulation performance, we have developed a simulation model which calculates the amount of light extracted from a blue light-emitting die depending on the shape and on the size of its encapsulation.

We have used the Zemax software, which is based on the Monte-Carlo ray-tracing. The typical LED COB geometry includes a $1 \times 1 \mathrm{~mm}^{2}$ GaN LED die (refractive index: $n=2.42$ for a $450 \mathrm{~nm}$ incident wavelength) on a large board. Then, the die emission has been modelled as a lambertian intensity distribution. The light-emitting wavelength has been fixed equal to $450 \mathrm{~nm}$, which is close to the typical wavelength of Royal Blue LEDs. For simplicity reasons, we have also chosen not to take into account the die roughness in these simulations. As will be detailed later, this does not impact the trend of the results, but it implies to carefully analyse them. Then, the board has been considered as a reflective element. In order to reproduce the behaviour of the printed circuit board (PCB) which will be used for the comparative experimental tests and which comprises a white solder mask, we have fixed its reflectivity to $70 \%$ with a surface lambertian diffusion. Finally, this module has been covered with a silicone encapsulation ( $n=1.4$ for a $450 \mathrm{~nm}$ incident wavelength), whose shape and size have been modified as depicted in Figure 1. $H$ is the height, and $R$ is the radius of the encapsulation.
The encapsulation shape has been set as half-ellipsoidal, with an aspect ratio "height above radius" $H / R$ varying from 0.3 (flat encapsulation) to 1.7 (oblong encapsulation). Thus, depending on the shape, the volume of the encapsulation varies accordingly from about $1 \mathrm{~mm}^{3}$ to $70 \mathrm{~mm}^{3}$, which allows observing simultaneously the effect of the shape and of the volume on the module optical characteristics.

Regarding the simulation analysis, this model does not take into account the die roughness as stated above. This does not significantly change the results since both the LED plane surface and a roughened surface have a lambertian distribution. However, it implies that the analysis has to be done by only comparing the results among themselves. Thus, we have normalized the light extraction values which have been obtained through the simulations as follows:

$$
\eta_{\text {extraction }}=\frac{\eta-\eta_{0}}{\eta_{0}}
$$

where $\eta$ is the light extraction efficiency of the considered geometry and $\eta_{0}$ is the light extraction efficiency of a reference case. This reference has been arbitrarily chosen to be a hemispherical encapsulation with a radius $R$ of $1.4 \mathrm{~mm}$.

Then, we have plotted this calculated gain or loss in light extraction $\eta_{\text {extraction }}$ depending on the volume and on the aspect ratio of the encapsulation. The corresponding results are given in Figure 2.

First, the graph clearly evidences that, due to a high amount of total internal reflexions within the encapsulation, a flat encapsulation shape $(H / R<0.7)$ ever provides a bad light extraction. This is in agreement with previous works [8] which were done for packaged LEDs, where it was shown that a hemispherical dome gave better extraction results than a flat or convex encapsulation shape.

Our simulation results also indicate that, above an aspect ratio $H / R$ of 0.7 , the shape of the encapsulation has a small impact on the light extraction and that even an oblong shape may provide an efficient LED COB module. This was also observed in recent works [12] on silicon-based LED packaging. Indeed, above a certain aspect ratio, the ray incident angles at the silicone-air interface are small enough to limit the total internal reflexions. Thus, these rays will always be able to escape from the encapsulation, maintaining the light extraction quite stable. 


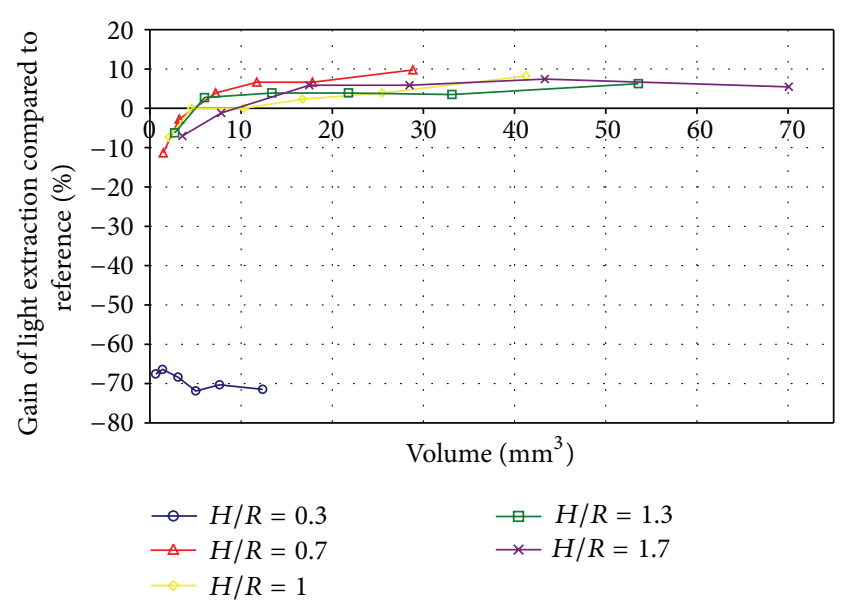

FIGURE 2: Variation of the light extraction of a LED COB module, depending on the volume and on the aspect ratio of the LED die silicone encapsulation (simulation results).

Then, regarding the impact of the encapsulation volume, previous works have shown that the light extraction stabilized from an encapsulation radius larger than $1.5 r$, where $r$ is the die size [10]. In our model configuration, the die size is $r=1 \mathrm{~mm}$. Thus, a hemispherical encapsulation such as $R=1.5 r$ approximately represents a volume of $7 \mathrm{~mm}^{3}$. Our simulations are also effectively in line with these results. More generally, our computations evidence that, above a silicone volume of 5 to $10 \mathrm{~mm}^{3}$, the light extraction efficiency of the $\mathrm{COB}$ module remains quite stable and rather independent of the encapsulation shape. However, for small silicone volumes, the hemispherical encapsulation shape is the most efficient geometry.

Finally, we have studied the impact of the LED COB encapsulation shape on the light intensity distribution. By inserting a far-field intensity detector in the simulation model, we have collected the angular flux emitted by the COB geometry. The light intensity distribution is plotted in Figure 3 for an encapsulation radius of $2.3 \mathrm{~mm}$.

As it is currently done for packaged LED components, the graph evidences that giving a specific shape to the LED COB encapsulation allows beam shaping. Indeed, inserting an oblong-shape encapsulation implies that light rays impacting the silicone-air interface will mainly be extracted along small angles. This will lead to a sharp and narrow intensity distribution, as it is depicted by the simulation curve representing the high aspect ratio $H / R=1.7$. On the contrary, making a hemispherical or flat encapsulation favours light angular dispersion at the silicone-air interface, which will provide a rather lambertian distribution.

These theoretical studies indicate that the optimal optical performances for a LED COB module will be obtained with a geometry combining a rather high encapsulation volume (larger than $5 \mathrm{~mm}^{3}$ ) and a hemispherical shape. An oblong shape may also be considered for applications which require strong narrow beams.

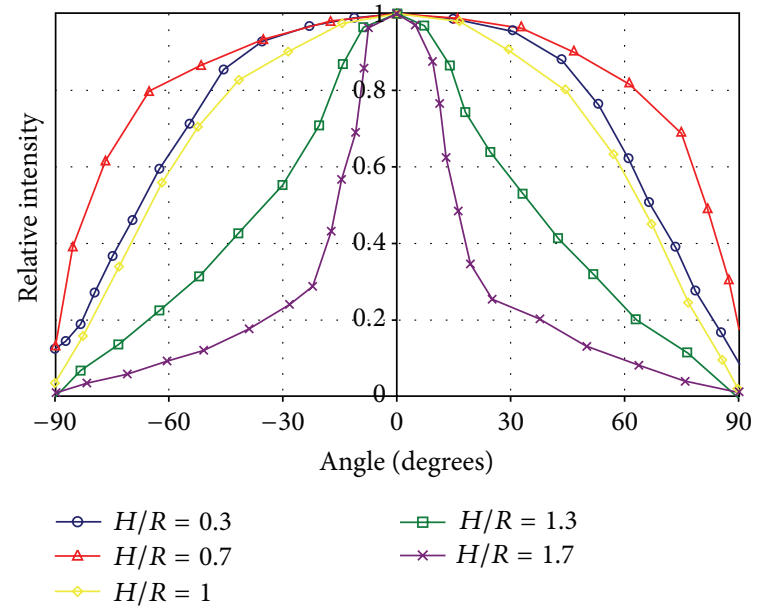

FIGURE 3: Light intensity distributions provided by a LED COB module depending on the shape of its encapsulation (simulation results). The encapsulation radius is $2.3 \mathrm{~mm}$.

\section{Experiments}

In order to validate the simulation results which have been presented above and to evaluate the potential of such encapsulation shapes and volumes for improving the performances of LED COB modules, we have mounted several LED dies in a COB configuration. Then, we have performed different tests on these modules to check their optical characteristics. A top view of one of these modules (microscopic imaging) is shown in Figure 4.

The modules include a SemiLEDS LED die of $1 \times$ $1 \mathrm{~mm}^{2}$ surface area with a typical dominant wavelength $\lambda_{\text {dom }}$ equal to $462.5 \mathrm{~nm}$ (reference SL-V-B40AC2, with a typical radiant flux at $350 \mathrm{~mA}$ of $350 \mathrm{~mW}$ ). The LED die has been mounted on a "star" PCB with a silver paste adhesive (Epoxy Technology, reference EPOTEK H20E-HC).

Encapsulation was carried out using a dispensing process with silicone from NuSil, having a refractive index $n=1.4$ for blue-range incident wavelengths. Volume and shape have been adjusted by varying the dispensing parameters.

Finally, we have used a 6 " integrating sphere coupled to a spectrophotometer from LabSphere (SphereOptics) to measure the radiant flux of these encapsulated modules. In order to be able to compare the experimental results with the ray-tracing simulations which were detailed in the previous section, we have followed the same analysis procedure (see (1)). First, we have calculated the light extraction of each module by doing the ratio between the radiant fluxes emitted by the system after and before encapsulation. Then, we have chosen a sample, which presents a hemispherical encapsulation of $2 \mathrm{~mm}$ radius, and we have set its light extraction value $\eta_{0}$ as reference for the others. Finally, we have normalized all of the sample extraction values to this reference case, and we have plotted the graph which is given in Figure 5. It has to be noted that, due to the different geometries of the two reference samples which have been taken for analysing the experimental and the simulation results (encapsulation radius of $2 \mathrm{~mm}$ versus $1.4 \mathrm{~mm}$ for the simulations), the 


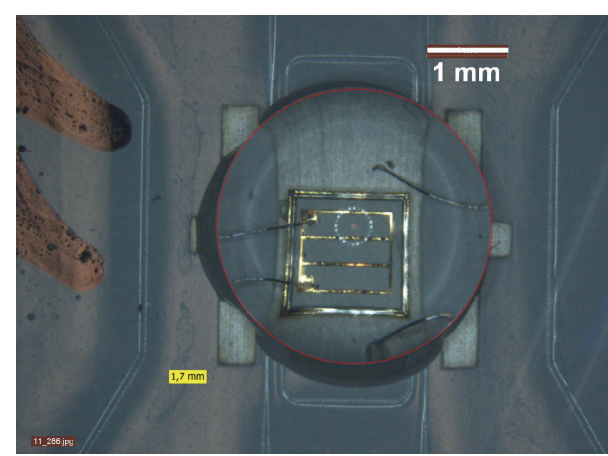

FIgURE 4: Top view of one LED COB module.

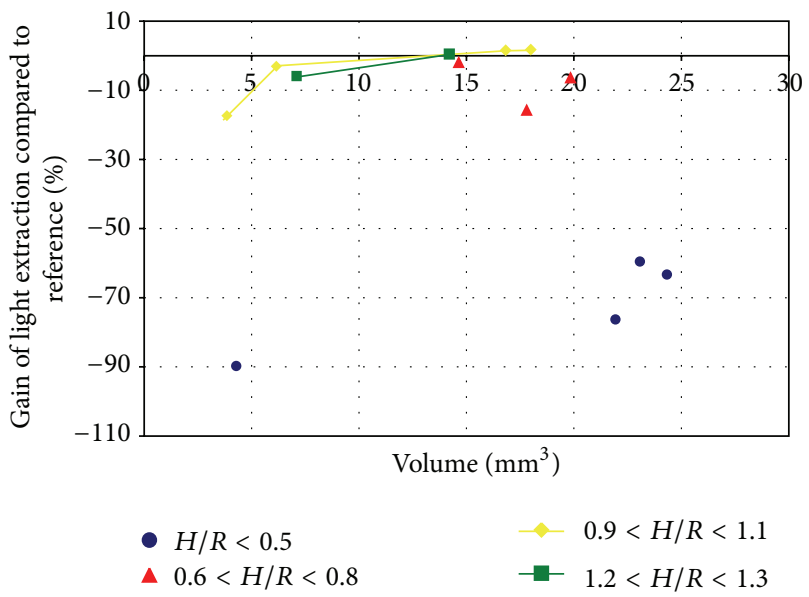

FIGURE 5: Gain of light extraction as a function of the encapsulation volume for various $H / R$ ratio (experimental results).

maximal gain of light extraction $\eta_{\text {extraction }}$ is different between these two graphs.

First, the graph confirms the simulation results which stipulated that a flat encapsulation never provides a good light extraction. Whatever the encapsulation volume is, the samples which present an aspect ratio $H / R$ smaller than 0.6 all lead to a poor light extraction. In addition, the absolute values of light extraction measured for these samples (not shown in the graph) also indicate that the radiant flux emitted by some of these modules is even lower than the initial flux of $350 \mathrm{~mW}$ emitted by the LED die without silicone encapsulation.

Then, the measurement dispersion is also larger than the simulated one. This is mainly due to the fact that the encapsulation shapes are not always ellipsoidal as it was modelled in the simulations. Indeed, we found that some of them present a ball-shape (diameter at the contact interface of the PCB lower than the encapsulation diameter). Such shapes, which appear to be a consequence of our manual dispense process, may effectively influence the light extraction efficiency of the COB modules. In order to check this phenomenon, we have chosen two of our samples, and we have performed additional simulations by modelling their real encapsulation shapes. The results are summarized in Table 1 . The results of the simulations done with ellipsoidal encapsulation shapes of similar volumes are also given. For comparison, the light
TABLE 1: Comparative light extraction efficiencies for COB modules, depending on their encapsulation shapes (ellipsoidal or ball-shape).

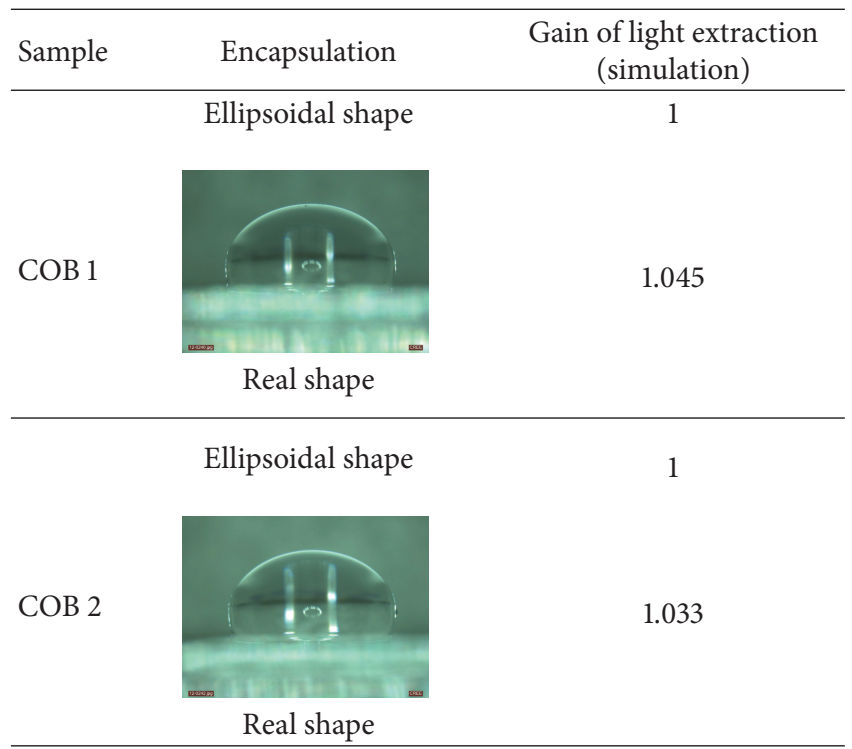

extraction obtained with these ellipsoidal encapsulations was arbitrarily normalized to 1 , and it has been taken as reference for the simulations of the ball-shape encapsulations.

These simulations evidence that ball shapes improve the light extraction efficiency of several percents compared to an ellipsoidal encapsulation of similar volume. Thus, an encapsulation shape variation may effectively be at the origin of the measurement dispersion compared to simulation results.

Nevertheless, these light extraction measurements generally show the same trend than the one which was observed with the simulations. For instance, when considering the small-volume encapsulations, the light extraction rather increases with the silicone volume.

Finally, among the different modules which were realized, the maximal light extraction is obtained for a rather hemispherical encapsulation, with a volume of about $15 \mathrm{~mm}^{3}$. This is in line with the simulation results, which predicted a stabilization of the light extraction at its optimum with encapsulation volumes larger than $10 \mathrm{~mm}^{3}$. We have measured the radiant flux emitted by this module: we found a light extraction value of about $30 \%$ compared to the radiant flux emitted by LED die without silicone, which is of the same order than the light extraction gains which have been measured for silicone packaged LEDs [13]. Thus, this confirms the potential of $\mathrm{COB}$ technology for providing highefficient LED light sources.

In order to check the impact of the encapsulation shape on the module intensity distribution, we have also performed angular measurements with a LED spectrogoniometer to obtain the light intensity distribution of the modules depending on their encapsulation aspect ratio. The results are shown in Figure 6.

This graph confirms experimentally the phenomena which were observed in simulations. The modules which were encapsulated with an oblong encapsulation shape $(H / R>1)$ 


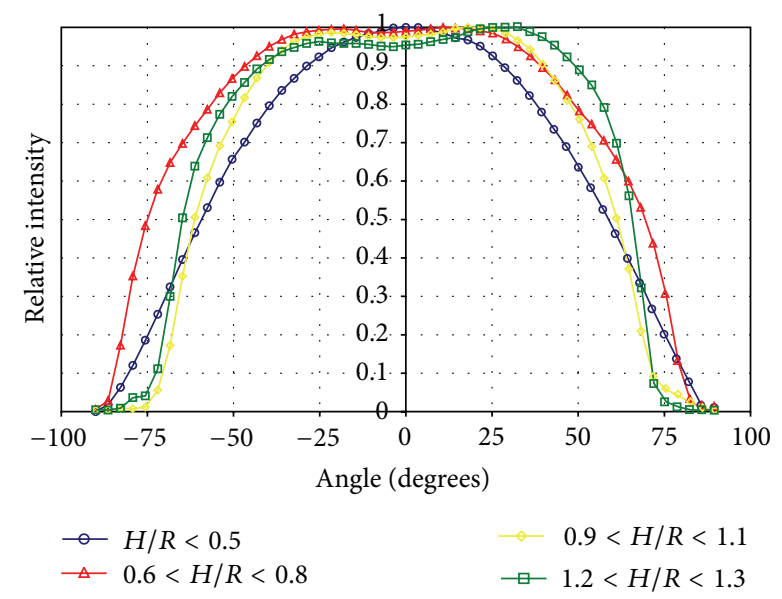

FIGURE 6: Light intensity distributions provided by a LED COB module depending on the shape of its encapsulation (experimental results).

provide a sharper and narrower distribution than the modules covered with a lower aspect ratio encapsulation: this evidences that light extraction at small exit angles is favoured. As theoretically expected, the hemispherical and encapsulation shapes rather provide lambertian distributions.

\section{Conclusions}

In this paper, we have studied theoretically and experimentally the impact of the encapsulation shape and the volume of a LED COB module on its performances, in terms of both light extraction and intensity distribution. We have shown that flat encapsulation shapes never provide an efficient COB light source, while hemispherical encapsulation shapes lead to lambertian distributions with a light extraction gain which is rather independent of the encapsulation shape above volumes of $10 \mathrm{~mm}^{3}$. Despite providing more dispersed data due to the process parameter variations, the experiments confirmed the simulation results. Presenting up to a $30 \%$ encapsulation gain in light extraction, they evidenced that the optimal performances are obtained with a nearly hemispherical encapsulation, with a volume for a $1 \mathrm{~mm}^{2}$ LED die larger than 5 to $10 \mathrm{~mm}^{3}$. This work is seen to gain an insight into the potential of LED COB modules for providing high-efficient and custom light sources. Next studies will be devoted to apply these results to white modules and to optimize the insertion of phosphors particles within the encapsulation to obtain efficient white LED COB light sources.

\section{Acknowledgments}

The authors would like to gratefully acknowledge NEOLUX (La Chapelle-Vendômoise, France) and BEST (GevreyChambertin, France) for partially funding this study.

\section{References}

[1] F. M. Steranka, J. Bhat, D. Collins et al., "High power LEDstechnology status and market applications," Physica Status Solidi A, vol. 194, no. 2, pp. 380-388, 2002.
[2] P. Hartmann, F. P. Wenzl, C. Sommer et al., "White LEDs and modules in Chip-On-Board technology for general lighting," Proc. of SPIE, vol. 6337, Article ID 63370I, 2006.

[3] E. F. Schubert, Light-Emitting Diodes, Cambridge University Press, 2006.

[4] T. Fujii, Y. Gao, R. Sharma, E. L. Hu, S. P. DenBaars, and S. Nakamura, "Increase in the extraction efficiency of GaN-based light-emitting diodes via surface roughening," Applied Physics Letters, vol. 84, no. 6, pp. 855-857, 2004.

[5] J. H. Lee, J. T. Oh, S. B. Choi, J. G. Woo, S. Y. Lee, and M. B. Lee, "Extraction-efficiency enhancement of InGaN-based vertical LEDs on hemispherically patterned sapphire," Physica Status Solidi C, vol. 4, no. 7, pp. 2806-2809, 2007.

[6] C. Nuese, J. Tietjen, J. Gannon, and H. Gossenberger, "Optimization of electroluminescent efficiencies for vapor-grown GaAsl-xPx diodes," Journal of The Electrochemical Society, vol. 116, no. 2, pp. 248-253, 1969.

[7] F. W. Mont, J. K. Kim, M. F. Schubert, H. Luo, E. F. Schubert, and R. W. Siegel, "High refractive index nanoparticle-loaded encapsulants for light-emitting diodes," in Light-Emitting Diodes: Research, Manufacturing, and Applications XI, Procceedings of SPIE, January 2007.

[8] J. K. Kim, H. Luo, E. F. Schubert, J. Cho, C. Sone, and Y. Park, "Strongly enhanced phosphor efficiency in GaInN white light-emitting diodes using remote phosphor configuration and diffuse reflector cup," Japanese Journal of Applied Physics, vol. 44, no. 20-23, pp. L649-L651, 2005.

[9] H. Luo, J. K. Kim, E. F. Schubert, J. Cho, C. Sone, and Y. Park, "Analysis of high-power packages for phosphor-based whitelight-emitting diodes," Applied Physics Letters, vol. 86, no. 24, Article ID 243505, pp. 1-3, 2005.

[10] H. C. Chen, K. J. Chen, C. H. Wang et al., "A novel randomly textured phosphor structure for highly efficient white lightemitting diodes," Nanoscale Research Letters, vol. 7, article 188, 2012.

[11] I. Moreno, D. Bermúdez, and M. Avendaño-Alejo, "Lightemitting diode spherical packages: an equation for the light transmission efficiency," Applied Optics, vol. 49, no. 1, pp. 12-20, 2010.

[12] B. Cao, S. Yu, H. Zheng, and S. Liu, "Silicon-based system in packaging for light emitting diodes," in Proceedings of the IEEE 62nd Electronic Components and Technology Conference, pp. 1267-1272, 2012.

[13] S. Liu and X. Luo, LED Packaging for Lighting Applications, Chemical Industry Press, 2011. 

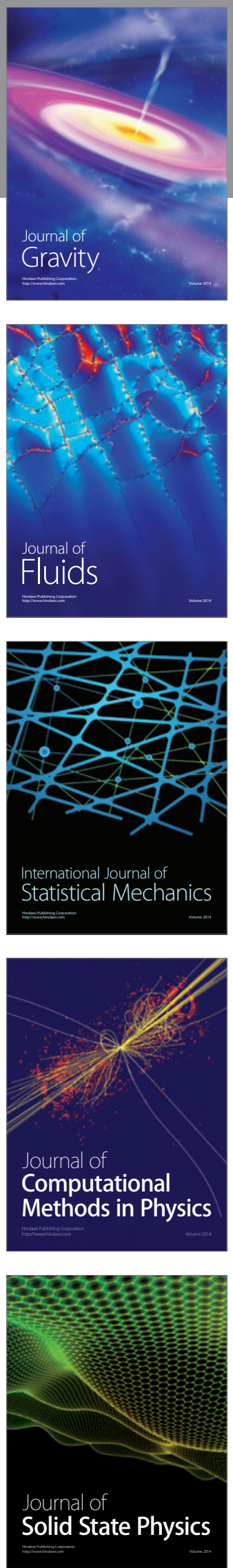

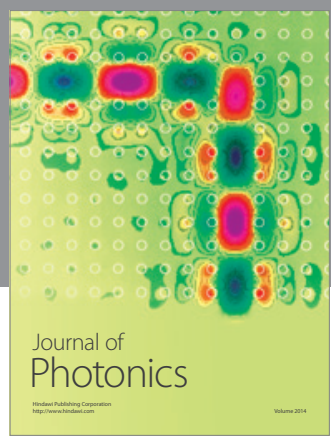

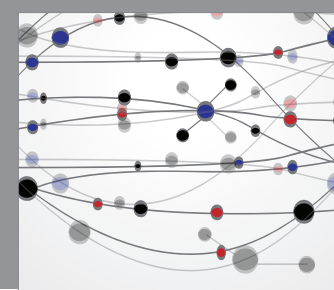

The Scientific World Journal

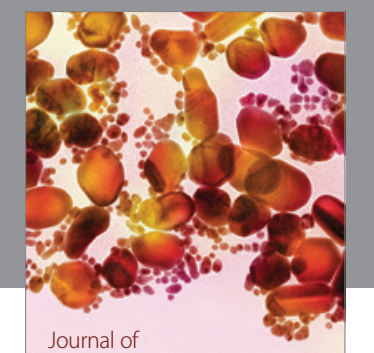

Soft Matter
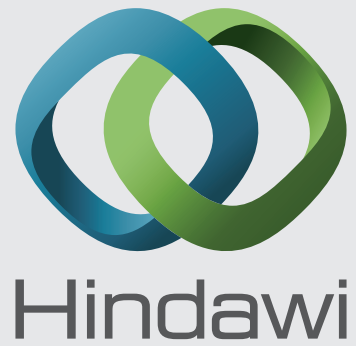

Submit your manuscripts at

http://www.hindawi.com
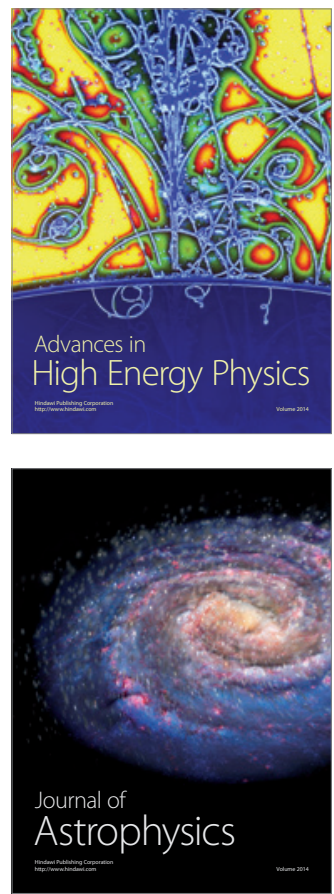
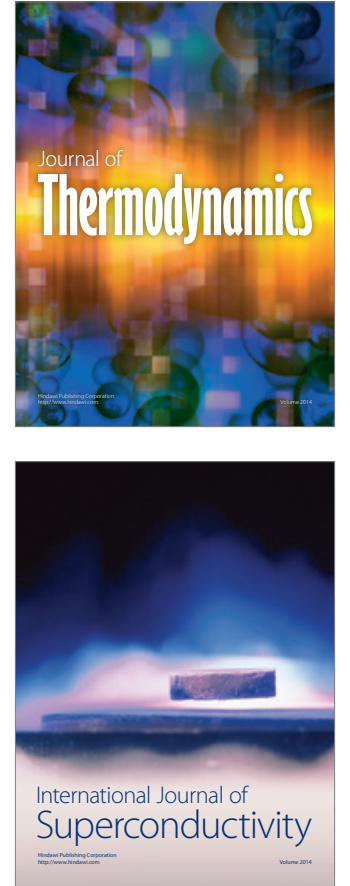
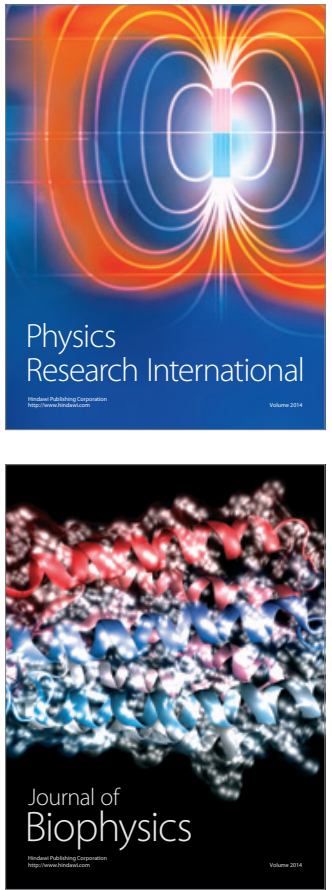
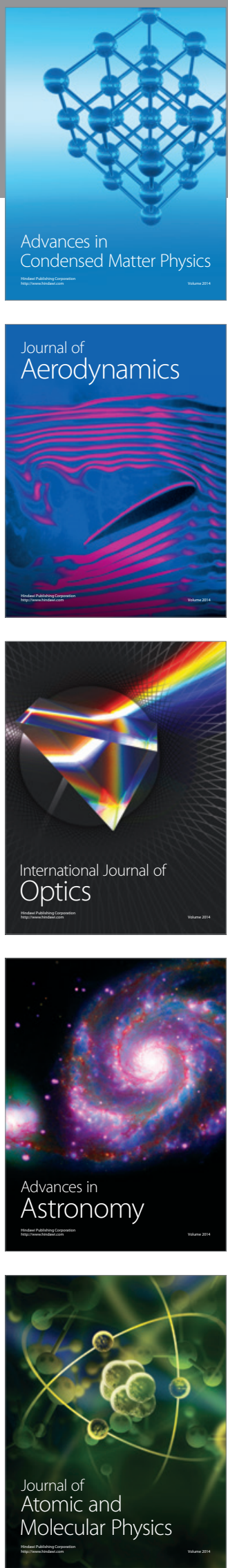